\title{
ON THE TIME BEHAVIOUR OF OKAZAKI FRAGMENTS
}

\author{
KRZYSZTOF BARTOSZEK * AND \\ WOJCIECH BARTOSZEK, ${ }^{* * *}$ Gdańsk University of Technology
}

\begin{abstract}
We find explicit analytical formulae for the time dependence of the probability of the number of Okazaki fragments produced during the process of DNA replication. This extends a result of Cowan on the asymptotic probability distribution of these fragments.
\end{abstract}

Keywords: DNA replication; quasi-renewal equation; Okazaki fragment

2000 Mathematics Subject Classification: Primary 60K05

Secondary 92B05; 92C45

In a simplified model of DNA replication, Cowan [2] obtained an asymptotic probability distribution for the number of small fragments of DNA produced when the process attains equilibrium. Such fragments are called Okazaki fragments. The reader is referred to [2]-[5] for biological background and details. Let us denote by $N_{t}(\omega)$ the number of Okazaki fragments at the instant $t \geq 0$. This is not a deterministic function, but rather a stochastic process with nonnegative (integer) values. Let $g_{i}(t)=\mathrm{P}\left(N_{t}=i\right)$. Assuming that so-called 'primers' appear according to a Poisson process with intensity $\lambda$, it can be proved (see [2], [3], and [6]) that the functions $g_{i}, i=0,1, \ldots$, satisfy the following system of (quasi-renewal) equations:

$$
\begin{aligned}
& g_{0}(t)=\mathrm{e}^{-\lambda t}+\int_{0}^{a t} g_{0}(t-y) \lambda \mathrm{e}^{-\lambda y} \mathrm{~d} y, \\
& g_{i}(t)=h_{i}(t)+\int_{0}^{a t} g_{i}(t-y) \lambda \mathrm{e}^{-\lambda y} \mathrm{~d} y, \quad i=1,2, \ldots
\end{aligned}
$$

(Readers unfamiliar with the concept of a primer are referred to [3] or [5] for a brief introduction.) The value of the constant $a, 0<a<1$, follows from the model and the functions $h_{i}$, $i=0,1, \ldots$, are as follows:

$$
h_{i}(t)= \begin{cases}\mathrm{e}^{-\lambda t}, & i=0, \\ \int_{a t}^{t} g_{i-1}(t-y) \lambda \mathrm{e}^{-\lambda y} \mathrm{~d} y, & i=1,2,3, \ldots\end{cases}
$$

A natural question arises as to whether such a system has a (unique) solution. If it does we may try to find formulae for the $g_{i}$. In his approach in [2], Cowan used the method developed earlier by Piau [6] in his studies of quasi-renewal equations and presented recurrence relationships for $g_{i}=\lim _{t \rightarrow \infty} g_{i}(t), i=0,1, \ldots$ It appears that the $g_{i}$ form a probabilistic distribution on

Received 11 November 2005; revision received 6 January 2006.

* Postal address: Department of Mathematics, Gdańsk University of Technology, ul. Narutowicza 11/12, 80-952

Gdańsk Wrzeszcz, Poland.

** Email address: bartowk@mifgate.mif.pg.gda.pl 
the set of nonnegative integers. In proving that the above system has a unique solution, Cowan considered the functions $g_{i}(t)$ to be integrable on each compact subset of $\mathbb{R}_{+}$. By applying the Laplace transform to $g_{i}(t)$ and using the Euler identity (see [1, p. 19]), Cowan finally calculated the generating function, the first moment, and the variance of the limit distribution $g_{i}$.

In our approach the $g_{i}(t)$ are considered to be bounded, continuous functions on $\mathbb{R}_{+}$. We directly prove the existence and uniqueness of such solutions and, as a side effect, obtain explicit formulae for the $g_{i}(t)$. By $C_{\mathrm{B}}$ we denote the Banach lattice of all bounded, continuous, realvalued functions on $\mathbb{R}_{+}$equipped with the supremum norm $\|f\|_{\text {sup }}=\sup _{t \in \mathbb{R}_{+}}|f(t)|$. We also introduce the Banach lattices $C_{\mathrm{B}, u}$ of all real-valued, continuous functions $f$ on finite intervals $[0, u], u>0$, with the same supremum norm (restricted to $t \in[0, u]$ ). Given a function $f \in C_{\mathrm{B}}$, we define

$$
R(f)(t)=\int_{0}^{a t} f(t-s) \lambda \mathrm{e}^{-\lambda s} \mathrm{~d} s .
$$

Clearly $R$ is a positive, linear operator on $C_{\mathrm{B}}$. It is not hard to see that

$$
R\left(\mathbf{1}_{[0, u]} f\right)(t)=\mathbf{1}_{[0, u]}(t) R(f)(t) .
$$

In other words, $R$ leaves $C_{\mathrm{B}, u}$ invariant and its restriction $R \uparrow C_{\mathrm{B}, u}$ may therefore be simply denoted $R$. Note that the operator norm of this restriction is $\left\|R \uparrow C_{\mathrm{B}, u}\right\|=1-\mathrm{e}^{-a u}$.

Given a function $h \in C_{\mathrm{B}}$, we define an affine operator

$$
T_{h}: C_{\mathrm{B}} \rightarrow C_{\mathrm{B}} \quad \text { by } \quad T_{h}(f)(t)=h+R(f)(t) .
$$

We note that $T_{h}$ also acts on $C_{\mathrm{B}, u}$ and that, for all $f_{1}, f_{2} \in C_{\mathrm{B}, u}$,

$$
\left\|T_{h}\left(f_{1}\right)-T_{h}\left(f_{2}\right)\right\|_{\text {sup }} \leq\left(1-\mathrm{e}^{-a u}\right)\left\|f_{1}-f_{2}\right\|_{\text {sup }} .
$$

By the Banach fixed-point theorem,

$$
T_{h}^{n}(f) \rightarrow f_{*, u} \quad \text { uniformly on }[0, u],
$$

where $f_{*, u}$ is a unique fixed-point of $T_{h} \uparrow C_{\mathrm{B}, u}$. Clearly there exists a unique $f_{*} \in C_{\mathrm{B}}$ (of course the limit depends on the control function $h$ ) such that $f_{*, u}=f_{*} \uparrow[0, u]$ and, moreover, for every $f \in C_{\mathrm{B}}, T^{n}(f) \rightarrow f_{*}$ uniformly on every compact subset of $R_{+}$. We easily find that

$$
T_{h}^{n}(f)(t)=\sum_{k=0}^{n-1} R^{k}(h)(t)+R^{n}(f)(t) .
$$

Notice that if we let $h=\mathrm{e}^{-\lambda t}$ then $g_{0}=f_{*}$.

We have just proved that the solution to the equation

$$
g_{0}(t)=\mathrm{e}^{-\lambda t}+\int_{0}^{a t} g_{0}(t-y) \lambda \mathrm{e}^{-\lambda y} \mathrm{~d} y
$$

does exist and is unique. Moreover, it may be obtained as the $\operatorname{limit}_{n \rightarrow \infty} \lim _{\mathrm{e}^{-\lambda t}}^{n}(f)$, where $f \in C_{\mathrm{B}}$ is arbitrary. Clearly, for each $f \in C_{\mathrm{B}}$, we have $\left\|R^{n}(f)\right\|_{\text {sup }} \rightarrow 0$. The following is a similar result.

Lemma 1. For each $i=0,1, \ldots$, the only solution to (1) has the form $g_{i}=\sum_{k=0}^{\infty} R^{k} h_{i}$, where the series converges uniformly on every compact subset of $\mathbb{R}_{+}$and is strictly increasing if we start with a positive function $f \in C_{\mathrm{B}}$. 
The next lemma is a step towards finding explicit solutions to these equations. Its proof is omitted, as it is a straightforward exercise.

Lemma 2. For any nonnegative integer $k$ and nonnegative real numbers $\alpha$ and $\lambda$,

$$
R^{k}\left(t^{\alpha} \mathrm{e}^{-\lambda t}\right)=\lambda^{k}\left(\prod_{j=1}^{k} \frac{1-b^{\alpha+j}}{\alpha+j}\right) t^{\alpha+k} \mathrm{e}^{-\lambda t}
$$

By substituting $\alpha=0$ into this we obtain the following corollary.

Corollary 1. For every $k=0,1, \ldots$, we have

$$
R^{k}\left(\mathrm{e}^{-\lambda t}\right)=\prod_{j=1}^{k}\left(1-b^{j}\right) \frac{(\lambda t)^{k}}{k !} \mathrm{e}^{-\lambda t} .
$$

We are now in a position to provide an explicit formula for the function $g_{0}$ and its limit at infinity. It should be noted that (3) has appeared before, in [2].

Proposition 1. We have

$$
g_{0}(t)=\sum_{k=0}^{\infty} \prod_{j=1}^{k}\left(1-b^{j}\right) \frac{(\lambda t)^{k}}{k !} \mathrm{e}^{-\lambda t}
$$

and

$$
g_{0}=\lim _{t \rightarrow \infty} g_{0}(t)=\prod_{j=1}^{\infty}\left(1-b^{j}\right) .
$$

Proof. Equation (2) is a direct application of Corollary 1 and Lemma 1. The second formula follows from two observations: the product $\prod_{j=0}^{k}\left(1-b^{j}\right)$ decreases in $k$ to $\prod_{j=0}^{\infty}\left(1-b^{j}\right)$, and as $t \rightarrow \infty$ the Poisson measure

$$
p_{\lambda t}=\mathrm{e}^{-\lambda t} \sum_{k=0}^{\infty} \frac{(\lambda t)^{k}}{k !} \delta_{k}
$$

tends weakly to $\delta_{\infty}$, where $\delta_{k}$ denotes the Dirac delta measure.

The following theorem provides explicit formulae for the functions $g_{i}$ and $h_{i}$, for $i=$ $0,1,2, \ldots$

Theorem 1. We have

$$
g_{i}(t)=\sum_{n_{0}=0, \ldots, n_{i}=0}^{\infty} \frac{\prod_{j=1}^{n_{0}+\cdots+n_{i}+i}\left(1-b^{j}\right) \prod_{k=1}^{i} b^{n_{1}+\cdots+n_{k}+k}}{\prod_{k=1}^{i}\left(1-b^{n_{1}+\cdots+n_{k}+k}\right)} \frac{(\lambda t)^{n_{0}+\cdots+n_{i}+i}}{\left(n_{0}+\cdots+n_{i}+i\right) !} \mathrm{e}^{-\lambda t}
$$

and

$$
h_{i}(t)=\sum_{n_{1}=0, \ldots, n_{i}=0}^{\infty} \frac{\prod_{j=1}^{n_{1}+\cdots+n_{i}+i}\left(1-b^{j}\right) \prod_{k=1}^{i} b^{n_{1}+\cdots+n_{k}+k}}{\prod_{k=1}^{i}\left(1-b^{n_{1}+\cdots+n_{k}+k}\right)} \frac{(\lambda t)^{n_{1}+\cdots+n_{i}+i}}{\left(n_{1}+\cdots+n_{i}+i\right) !} \mathrm{e}^{-\lambda t} .
$$


Proof. We have already discussed the case $i=0$ (see Corollary 1). Applying the induction method, let us assume that the formula holds for $i-1$. Elementary calculus yields

$$
\begin{aligned}
\int_{a t}^{t}(t-s)^{n_{0}+\cdots+n_{i-1}+i-1} \mathrm{e}^{-\lambda(t-s)} \lambda \mathrm{e}^{-\lambda s} \mathrm{~d} s & =\lambda \int_{a t}^{t}(t-s)^{n_{0}+\cdots+n_{i-1}+i-1} \mathrm{e}^{-\lambda t} \mathrm{~d} s \\
& =\left.\lambda \mathrm{e}^{-\lambda t}\left(-\frac{(t-s)^{n_{0}+\cdots+n_{i-1}+i}}{\left(n_{0}+\cdots+n_{i-1}+i\right) !}\right)\right|_{a t} ^{t} \\
& =\lambda \mathrm{e}^{-\lambda t} \frac{(t-a t)^{n_{0}+\cdots+n_{i-1}+i}}{\left(n_{0}+\cdots+n_{i-1}+i\right) !} \\
& =\lambda \mathrm{e}^{-\lambda t} \frac{b^{n_{0}+\cdots+n_{i-1}+i} t^{n_{0}+\cdots+n_{i-1}+i}}{\left(n_{0}+\cdots+n_{i-1}+i\right) !} .
\end{aligned}
$$

In order to keep our proof compact we make the following abbreviations:

$$
\begin{gathered}
L_{i}=\left(\prod_{j=1}^{n_{0}+\cdots+n_{i}+i}\left(1-b^{j}\right)\right) \prod_{k=1}^{i} \frac{b^{n_{1}+\cdots+n_{k}+k}}{1-b^{n_{1}+\cdots+n_{k}+k}} \frac{1}{\left(n_{0}+\cdots+n_{i}+i\right) !}, \\
\Lambda_{i}(t)=\frac{(\lambda t)^{n_{0}+\cdots+n_{i-1}+i}}{\left(n_{0}+\cdots+n_{i-1}+i\right) !} \mathrm{e}^{-\lambda t} .
\end{gathered}
$$

Now

$$
\begin{aligned}
& h_{i}(t) \\
& =\int_{a t}^{t} g_{i-1}(t-s) \lambda \mathrm{e}^{-\lambda s} \mathrm{~d} s \\
& =\int_{a t}^{t} \sum_{n_{0}=0, \ldots, n_{i-1}=0}^{\infty} L_{i-1} \frac{\lambda^{n_{0}+\cdots+n_{i-1}+i-1}(t-s)^{n_{0}+\cdots+n_{i-1}+i-1}}{\left(n_{0}+\cdots+n_{i-1}+i-1\right) !} \mathrm{e}^{-\lambda(t-s)} \lambda \mathrm{e}^{-\lambda s} \mathrm{~d} s \\
& =\sum_{n_{0}=0, \ldots, n_{i-1}=0}^{\infty} L_{i-1} \frac{\lambda^{n_{0}+\cdots+n_{i-1}+i-1}}{\left(n_{0}+\cdots+n_{i-1}+i-1\right) !} \int_{a t}^{t}(t-s)^{n_{0}+\cdots+n_{i-1}+i-1} \mathrm{e}^{-\lambda(t-s)} \lambda \mathrm{e}^{-\lambda s} \mathrm{~d} s \\
& =\sum_{n_{0}=0, \ldots, n_{i-1}=0}^{\infty} L_{i-1} \frac{\lambda^{n_{0}+\cdots+n_{i-1}+i-1}}{\left(n_{0}+\cdots+n_{i-1}+i-1\right) !} \lambda \mathrm{e}^{-\lambda t} \frac{b^{n_{0}+\cdots+n_{i-1}+i} t^{n_{0}+\cdots+n_{i-1}+i}}{\left(n_{0}+\cdots+n_{i-1}+i\right) !} \\
& =\sum_{n_{0}=0, \ldots, n_{i-1}=0}^{\infty} L_{i-1} b^{n_{0}+\cdots+n_{i-1}+i} \frac{(\lambda t)^{n_{0}+\cdots+n_{i-1}+i}}{\left(n_{0}+\cdots+n_{i-1}+i\right) !} \mathrm{e}^{-\lambda t} \\
& =\sum_{n_{0}=0, \ldots, n_{i-1}=0}^{\infty} L_{i-1}\left(1-b^{n_{0}+\cdots+n_{i-1}+i}\right) \frac{b^{n_{0}+\cdots+n_{i-1}+i}}{1-b^{n_{0}+\cdots+n_{i-1}+i}} \frac{(\lambda t)^{n_{0}+\cdots+n_{i-1}+i}}{\left(n_{0}+\cdots+n_{i-1}+i\right) !} \mathrm{e}^{-\lambda t} \\
& =\sum_{n_{0}=0, \ldots, n_{i-1}=0}^{\infty} \prod_{j=1}^{n_{0}+\cdots+n_{i-1}+i}\left(1-b^{j}\right) \frac{\prod_{k=1}^{i-1} b^{n_{1}+\cdots+n_{k}+k}}{\prod_{k=1}^{i-1}\left(1-b^{n_{1}+\cdots+n_{k}+k}\right)} \frac{b^{n_{1}+\cdots+n_{i-1}+n_{0}+i}}{1-b^{n_{1}+\cdots+n_{i-1}+n_{0}+i}} \Lambda_{i}(t) .
\end{aligned}
$$


By renaming the index $n_{0}$ as $n_{i}$ in the above summation, we obtain

$$
h_{i}(t)=\sum_{n_{1}=0, \ldots, n_{i}=0}^{\infty} \prod_{j=1}^{n_{1}+\cdots+n_{i}+i}\left(1-b^{j}\right) \frac{\prod_{k=1}^{i} b^{n_{1}+\cdots+n_{k}+k}}{\prod_{k=1}^{i}\left(1-b^{n_{1}+\cdots+n_{k}+k}\right)} \frac{(\lambda t)^{n_{1}+\cdots+n_{i}+i}}{\left(n_{1}+\cdots+n_{i}+i\right) !} \mathrm{e}^{-\lambda t}
$$

Applying Lemmas 1 and 2 yields

$$
\begin{aligned}
g_{i}(t)= & \sum_{n_{0}=0}^{\infty} R^{n_{0}}\left(h_{i}\right)(t) \\
= & \sum_{n_{0}=0}^{\infty} \sum_{n_{1}=0, \ldots, n_{i}=0}^{\infty} \frac{\prod_{j=1}^{n_{1}+\cdots+n_{i}+i}\left(1-b^{j}\right) \prod_{k=1}^{i} b^{n_{1}+\cdots+n_{k}+k}}{\prod_{k=1}^{i}\left(1-b^{n_{1}+\cdots+n_{k}+k}\right)} \\
& \times R^{n_{0}}\left(\frac{(\lambda t)^{n_{1}+\cdots+n_{i}+i}}{\left(n_{1}+\cdots+n_{i}+i\right) !} \mathrm{e}^{-\lambda t}\right) \\
= & \sum_{n_{0}=0, \ldots, n_{i}=0}^{\infty} \frac{\prod_{j=1}^{n_{1}+\cdots+n_{i}+i}\left(1-b^{j}\right) \prod_{k=1}^{i} b^{n_{1}+\cdots+n_{k}+k}}{\prod_{k=1}^{i}\left(1-b^{n_{1}+\cdots+n_{k}+k}\right)} \frac{\lambda^{n_{1}+\cdots+n_{i}+i}}{\left(n_{1}+\cdots+n_{i}+i\right) !} \\
= & \sum_{n_{0}=0, \ldots, n_{i}=0}^{\infty} \frac{\prod^{n_{0}} \prod_{k=1}^{n_{0}} \frac{1-b^{n_{1}+\cdots+n_{i}+i+k}}{n_{1}+\cdots+n_{i}+i+k} t^{n_{0}+\cdots+n_{i}+i} \mathrm{e}^{-\lambda t}}{\prod_{k=1}^{i}\left(1-b^{n_{1}+\cdots+n_{k}+k}\right)} \frac{(\lambda t)^{n_{0}+\cdots+n_{i}+i}}{\left(n_{0}+\cdots+n_{i}+i\right) !} \mathrm{e}^{-\lambda t} .
\end{aligned}
$$

The results of the theorem now follow by induction.

The next theorem describes the asymptotics.

Theorem 2. For each $i>0$, we have

$$
\begin{aligned}
g_{i}=\lim _{t \rightarrow \infty} g_{i}(t) & =\prod_{j=1}^{\infty}\left(1-b^{j}\right) \sum_{n_{1}=0, \ldots, n_{i}=0}^{\infty} \prod_{k=1}^{i} \frac{b^{n_{1}+\cdots+n_{k}+k}}{1-b^{n_{1}+\cdots+n_{k}+k}} \\
& =\prod_{j=1}^{\infty}\left(1-b^{j}\right) \sum_{n_{1}=1, \ldots, n_{i}=1}^{\infty} \prod_{k=1}^{i} \frac{b^{n_{1}+\cdots+n_{k}}}{1-b^{n_{1}+\cdots+n_{k}}}
\end{aligned}
$$

Proof. Note that

$$
g_{i}(t)=\sum_{n_{1}=0, \ldots, n_{i}=0}^{\infty} \prod_{k=1}^{i} \frac{b^{n_{1}+\cdots+n_{k}+k}}{1-b^{n_{1}+\cdots+n_{k}}} \sum_{n_{0}=0}^{\infty} \prod_{j=1}^{n_{0}+\cdots+n_{i}+i}\left(1-b^{j}\right) \frac{(\lambda t)^{n_{0}+\cdots+n_{i}+i}}{\left(n_{0}+\cdots+n_{i}+i\right) !} \mathrm{e}^{-\lambda t} .
$$


Using the same argument as in the proof of Proposition 1 for fixed values $n_{1}, \ldots, n_{i} \geq 0$, we obtain

$$
\begin{aligned}
\lim _{t \rightarrow \infty} & \sum_{n_{0}=0}^{\infty} \prod_{j=1}^{n_{0}+\cdots+n_{i}+i}\left(1-b^{j}\right) \frac{(\lambda t)^{n_{0}+\cdots+n_{i}+i}}{\left(n_{0}+\cdots+n_{i}+i\right) !} \mathrm{e}^{-\lambda t} \\
& =\prod_{j=1}^{\infty}\left(1-b^{j}\right) \lim _{t \rightarrow \infty}\left(1-\sum_{k=0}^{n_{1}+\cdots+n_{i}+i-1} \frac{(\lambda t)^{k}}{k !} \mathrm{e}^{-\lambda t}\right) \\
& =\prod_{j=1}^{\infty}\left(1-b^{j}\right) .
\end{aligned}
$$

The claim (in its first form) now follows from the Lebesgue convergence theorem and the fact that the series

$$
\sum_{n_{1}=0, \ldots, n_{i}=0}^{\infty} \prod_{k=1}^{i} \frac{b^{n_{1}+\cdots+n_{k}+k}}{1-b^{n_{1}+\cdots+n_{k}}}
$$

converges absolutely. Changing each summation to start at 1 instead of 0 yields the claim in its second form.

An application of the geometric formula yields the following corollary.

Corollary 2. For each $i=1,2, \ldots$, we have

$$
g_{i}=\prod_{j=1}^{\infty}\left(1-b^{j}\right) \sum_{n_{1}=1, \ldots, n_{i}=1}^{\infty} \prod_{k=1}^{i} \sum_{l=1}^{\infty} b^{\left(n_{1}+\cdots+n_{k}\right) l} .
$$

We will reduce the above multiple series to a simpler recurrence expression. Note that

$$
\begin{aligned}
& \quad \sum_{n_{1}=1, \ldots, n_{i}=1}^{\infty} \frac{b^{n_{1}+\cdots+n_{i}}}{1-b^{n_{1}+\cdots+n_{i}}} \frac{b^{n_{2}+\cdots+n_{i}}}{1-b^{n_{2}+\cdots+n_{i}}} \cdots \frac{b^{n_{i}}}{1-b^{n_{i}}} \\
& \quad=\sum_{m_{i}=i}^{\infty} \frac{b^{m_{i}}}{1-b^{m_{i}}} \sum_{m_{i-1}=i-1}^{m_{i}-1} \frac{b^{m_{i-1}}}{1-b^{m_{i-1}}} \sum_{m_{i-2}=i-2}^{m_{i-1}-1} \frac{b^{m_{i-2}}}{1-b^{m_{i-2}}} \cdots \sum_{m_{1}=1}^{m_{2}-1} \frac{b^{m_{1}}}{1-b^{m_{1}}} .
\end{aligned}
$$

For given natural numbers $i$ and $r, r \geq i$, we define

$$
\Psi_{i, r}(b)=\sum_{m_{i-1}=i-1}^{r-1} \frac{b^{m_{i-1}}}{1-b^{m_{i-1}}} \sum_{m_{i-2}=i-2}^{m_{i-1}-1} \frac{b^{m_{i-2}}}{1-b^{m_{i-2}}} \cdots \sum_{m_{1}=1}^{m_{2}-1} \frac{b^{m_{1}}}{1-b^{m_{1}}} .
$$

Clearly, for $s \geq i+1$, we have

$$
\Psi_{i+1, s}(b)=\sum_{r=i}^{s-1} \frac{b^{r}}{1-b^{r}} \Psi_{i, r}(b),
$$

where we have set $\Psi_{1, r}(b) \equiv 1$ for all $r \geq 1$. 


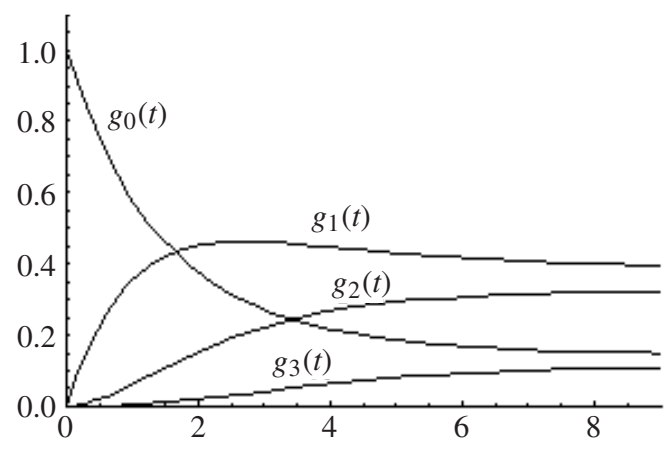

Figure 1.

We are now in a position to present the promised recursion formula for $g_{i}$.

Proposition 2. For each natural number $i$, we have

$$
g_{i}=\prod_{j=1}^{\infty}\left(1-b^{j}\right) \sum_{m=i}^{\infty} \frac{b^{m}}{1-b^{m}} \Psi_{i, m}(b) .
$$

Remark 1. In [2] another representation for $g_{i}$ can be found:

$$
g_{i}=\sum_{m=i}^{\infty}(-1)^{m-i}\left(\begin{array}{c}
m \\
i
\end{array}\right) \prod_{k=1}^{m} \frac{b^{k}}{1-b^{k}} .
$$

The above formulae were used to evaluate the values $g_{0}, \ldots, g_{10}$ for $\lambda=1$ and $b=0.6$ (programming in $\mathrm{C}$ ):

$$
\begin{array}{crl}
g_{0}=0.1431293315359, & g_{1}=0.3852183066464, \\
g_{2}=0.3269335487938, & g_{3}=0.1204847773561, \\
g_{4}=0.0220251599091, & g_{5}=0.0021441293616, \\
g_{6}=0.0001159472975, & g_{7}=0.0000035766460, \\
g_{8}=0.0000000640275, & g_{9}=0.0000000006727, \\
g_{10}=0.000000000004 .
\end{array}
$$

Furthermore, we include a diagram (see Figure 1), produced using MATHEMATICA ${ }^{\circledR}$, which contains sketches of the functions $g_{0}(t), g_{1}(t), g_{2}(t)$, and $g_{3}(t)$. We display them to give a general idea of what these functions look like: no formal numerical analysis or error evaluation was performed. As before, $\lambda=1$ and $b=0.6$.

To finish the paper we will prove that $\mathfrak{g}=\left\{g_{i}\right\}_{i=0}^{\infty}$ defines a probability distribution on the positive integers (i.e. that $\sum_{i=0}^{\infty} g_{i}=1$ ), and find its moments. For the convenience of the reader and completeness of the paper, we include all details (some ideas are adopted from [3] and [5]). Let us write

$$
n_{k}(t)=\mathrm{E}\left(N_{t}^{k}\right), \quad k=0,1, \ldots, t \geq 0 .
$$

Since $0^{0}=1$, we have $n_{0}(t) \equiv 1$. Clearly, for every $t$ and $k$, the moments $n_{k}(t)$ exist (notice that for a fixed $t \geq 0$ the process $N_{t}$ is dominated by the classical Poisson process). The existence 
of $\lim _{t \rightarrow \infty} n_{1}(t)<\infty$ implies that the distribution $\mathfrak{g}$ is nondegenerate (i.e. that $\sum_{i=0}^{\infty} g_{i}=1$ ). More generally,

$$
\lim _{t \rightarrow \infty} n_{k+1}(t)<\infty
$$

implies that the $k$ th moment of $\mathfrak{g}$ is finite. We will find a formula for $n_{k}(t)$. Our approach is direct and requires solving linear differential equations. Let $T$ denote the time we have to wait before the first primer appears. We begin (cf. [3]) with

$$
\begin{aligned}
n_{k}(t) & =\int_{0}^{a t} \mathrm{E}\left(N_{t}^{k} \mid T=s\right) \lambda \mathrm{e}^{-\lambda s} \mathrm{~d} s+\int_{a t}^{t} \mathrm{E}\left(N_{t}^{k} \mid T=s\right) \lambda \mathrm{e}^{-\lambda s} \mathrm{~d} s \\
& =\int_{0}^{a t} \mathrm{E}\left(N_{t-s}^{k}\right) \lambda \mathrm{e}^{-\lambda s} \mathrm{~d} s+\int_{a t}^{t} \mathrm{E}\left(N_{t-s}+1\right)^{k} \lambda \mathrm{e}^{-\lambda s} \mathrm{~d} s \\
& =\int_{0}^{a t} n_{k}(t-s) \lambda \mathrm{e}^{-\lambda s} \mathrm{~d} s+\int_{a t}^{t} \sum_{j=0}^{k}\left(\begin{array}{l}
k \\
j
\end{array}\right) n_{j}(t-s) \lambda \mathrm{e}^{-\lambda s} \mathrm{~d} s \\
& =\int_{0}^{a t} n_{k}(t-s) \lambda \mathrm{e}^{-\lambda s} \mathrm{~d} s+\int_{a t}^{t} n_{k}(t-s) \lambda \mathrm{e}^{-\lambda s} \mathrm{~d} s+\sum_{j=0}^{k-1}\left(\begin{array}{c}
k \\
j
\end{array}\right) \int_{a t}^{t} n_{j}(t-s) \lambda \mathrm{e}^{-\lambda s} \mathrm{~d} s \\
& =\int_{0}^{t} n_{k}(s) \lambda \mathrm{e}^{-\lambda t+\lambda s} \mathrm{~d} s+\sum_{j=0}^{k-1}\left(\begin{array}{c}
k \\
j
\end{array}\right) \int_{a t}^{t} n_{j}(t-s) \lambda \mathrm{e}^{-\lambda s} \mathrm{~d} s \\
& =\mathrm{e}^{-\lambda t} \int_{0}^{t} n_{k}(s) \lambda \mathrm{e}^{\lambda s} \mathrm{~d} s+\sum_{j=0}^{k-1}\left(\begin{array}{c}
k \\
j
\end{array}\right) \int_{a t}^{t} n_{j}(t-s) \lambda \mathrm{e}^{-\lambda s} \mathrm{~d} s .
\end{aligned}
$$

It follows from this representation that the functions $n_{k}$ (since they are measurable) belong to $C^{\infty}\left(\mathbb{R}_{+}\right)$. By differentiating both sides we obtain

$$
\begin{aligned}
n_{k}^{\prime}(t)= & -\lambda \mathrm{e}^{-\lambda t} \int_{0}^{t} n_{k}(s) \lambda \mathrm{e}^{\lambda s} \mathrm{~d} s+\mathrm{e}^{-\lambda t} n_{k}(t) \lambda \mathrm{e}^{\lambda t} \\
& +\sum_{j=0}^{k-1}\left(\begin{array}{l}
k \\
j
\end{array}\right)\left[n_{j}(0) \lambda \mathrm{e}^{-\lambda t}-a n_{j}(t-a t) \lambda \mathrm{e}^{-\lambda a t}+\int_{a t}^{t} n_{j}^{\prime}(t-s) \lambda \mathrm{e}^{-\lambda s} \mathrm{~d} s\right] .
\end{aligned}
$$

Note that $n_{j}(0)=1$ if $j=0$ and $n_{j}(0)=0$ for $j \geq 1$. It follows that

$$
\begin{aligned}
n_{k}^{\prime}(t)= & -\lambda \mathrm{e}^{-\lambda t} \int_{0}^{t} n_{k}(s) \lambda \mathrm{e}^{\lambda s} \mathrm{~d} s+\lambda n_{k}(t)+\lambda \mathrm{e}^{-\lambda t} \\
& +\sum_{j=0}^{k-1}\left(\begin{array}{l}
k \\
j
\end{array}\right)\left[\int_{a t}^{t} n_{j}^{\prime}(t-s) \lambda \mathrm{e}^{-\lambda s} \mathrm{~d} s-a n_{j}((1-a) t) \lambda \mathrm{e}^{-\lambda a t}\right] .
\end{aligned}
$$

Using (4), we obtain

$$
\mathrm{e}^{-\lambda t} \int_{0}^{t} n_{k}(s) \lambda \mathrm{e}^{\lambda s} \mathrm{~d} s=n_{k}(t)-\sum_{j=0}^{k-1}\left(\begin{array}{l}
k \\
j
\end{array}\right) \int_{a t}^{t} n_{j}(t-s) \lambda \mathrm{e}^{-\lambda s} \mathrm{~d} s
$$


Substituting this into (5) yields

$$
\begin{aligned}
n_{k}^{\prime}(t)= & -\lambda\left[n_{k}(t)-\sum_{j=0}^{k-1}\left(\begin{array}{l}
k \\
j
\end{array}\right) \int_{a t}^{t} n_{j}(t-s) \lambda \mathrm{e}^{-\lambda s} \mathrm{~d} s\right] \\
& +\lambda n_{k}(t)+\lambda \mathrm{e}^{-\lambda t}+\sum_{j=0}^{k-1}\left(\begin{array}{l}
k \\
j
\end{array}\right)\left[\int_{a t}^{t} n_{j}^{\prime}(t-s) \lambda \mathrm{e}^{-\lambda s} \mathrm{~d} s-a n_{j}((1-a) t) \lambda \mathrm{e}^{-\lambda a t}\right] \\
= & \lambda^{2} \sum_{j=0}^{k-1}\left(\begin{array}{l}
k \\
j
\end{array}\right) \int_{a t}^{t} n_{j}(t-s) \mathrm{e}^{-\lambda s} \mathrm{~d} s+\lambda \mathrm{e}^{-\lambda t} \\
& +\lambda \sum_{j=0}^{k-1}\left(\begin{array}{l}
k \\
j
\end{array}\right)\left[\int_{a t}^{t} n_{j}^{\prime}(t-s) \mathrm{e}^{-\lambda s} \mathrm{~d} s-a n_{j}(b t) \mathrm{e}^{-\lambda a t}\right] .
\end{aligned}
$$

Using the above recursion scheme and the induction principle, we easily obtain the following result.

Corollary 3. There exist constants $\beta_{k, j}>0, C_{k}>0$, and $\alpha_{k, j}$ and a natural number $L_{k}$ such that, for each $k \geq 0$, we have

$$
n_{k}(t)=\sum_{j=1}^{L_{k}} \alpha_{k, j} \mathrm{e}^{-\beta_{k, j} t}+C_{k}
$$

In particular,

$$
\lim _{t \rightarrow \infty} n_{k}(t)=\lim _{t \rightarrow \infty} \sum_{i=0}^{\infty} i^{k} g_{i}(t)=\sum_{i=0}^{\infty} i^{k} g_{i}=C_{k}<\infty
$$

(all moments of the asymptotic distribution $\mathfrak{g}$ are finite).

Setting $k=1$ in (6) yields

$$
n_{1}^{\prime}(t)=\lambda^{2} \int_{a t}^{t} \mathrm{e}^{-\lambda s} \mathrm{~d} s+\lambda \mathrm{e}^{-\lambda t}-a \lambda \mathrm{e}^{-\lambda a t}=\lambda(1-a) \mathrm{e}^{-\lambda a t}
$$

It follows that

$$
n_{1}(t)=\int \lambda(1-a) \mathrm{e}^{-\lambda a t} \mathrm{~d} t=-\frac{1-a}{a} \mathrm{e}^{-\lambda a t}+C .
$$

Clearly $C=(1-a) / a$, since $\lim _{t \rightarrow 0^{+}} n_{1}(t)=0$. As a result we obtain the next corollary.

Corollary 4. For all $t \geq 0$, we have $n_{1}(t)=(1-a)\left(1-\mathrm{e}^{-\lambda a t}\right) / a$. It follows that

$$
\mu=\sum_{i=0}^{\infty} i g_{i}=\lim _{t \rightarrow \infty} n_{1}(t)=\frac{1-a}{a} .
$$

In order to find the second moment and variance, we set $k=2$ in (6). After several elementary calculations, we obtain

$$
n_{2}^{\prime}(t)=\lambda \frac{(1-a)(2-a)}{a} \mathrm{e}^{-\lambda a t}-2 \lambda \frac{(1-a)^{2}}{a} \mathrm{e}^{-\lambda a(2-a) t} .
$$

Integrating the last equation and taking into account the fact that $n_{2}(0)=0$ for all $t \geq 0$ yields our final corollary. 


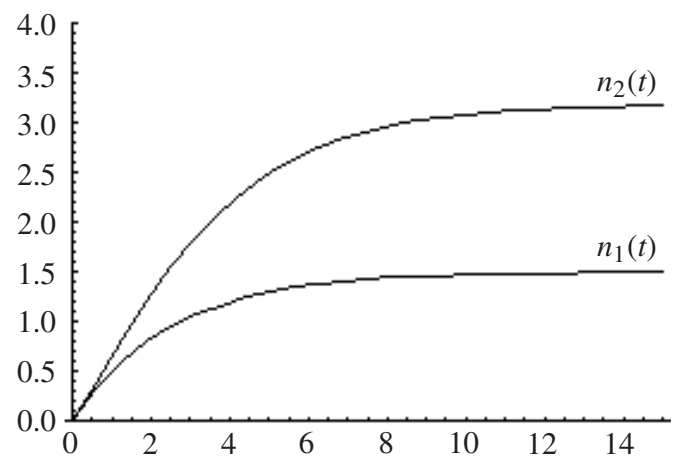

FigURE 2.

Corollary 5. We have

$$
n_{2}(t)=\frac{2(1-a)^{2}}{a^{2}(2-a)} \mathrm{e}^{-\lambda a(2-a) t}-\frac{(1-a)(2-a)}{a^{2}} \mathrm{e}^{-\lambda a t}+\frac{(1-a)\left(a^{2}-2 a+2\right)}{a^{2}(2-a)} .
$$

It follows that

$$
\lim _{t \rightarrow \infty} n_{2}(t)=\frac{(1-a)\left(a^{2}-2 a+2\right)}{a^{2}(2-a)}
$$

In particular,

$$
\operatorname{var}(\mathfrak{g})=\lim _{t \rightarrow \infty}\left(n_{2}(t)-n_{1}(t)^{2}\right)=\frac{1-a}{1-(1-a)^{2}} .
$$

In Figure 2 we display the graphs of the functions $n_{1}(t)$ and $n_{2}(t)$ for $\lambda=1$ and $a=1-b=0.4$.

\section{References}

[1] Andrews, G. (1976). The Theory of Partitions (Encyclopaedia Math. Appl. 2). Addison-Wesley, Reading, MA.

[2] CowAn, R. (2001). A new discrete distribution arising in a model of DNA replication. J. Appl. Prob. 38, 754-760.

[3] Cowan, R. (2003). Stochastic models for DNA replication. In Handbook of Statistics, Vol. 21, eds D. N. Shanbhag et al., North-Holland, Amsterdam, pp. 137-166.

[4] Cowan, R. AND ChiU, S. N. (1994). A stochastic model of fragment formation when DNA replicates. J. Appl. Prob. 31, 301-308.

[5] Lachal, A. (2003). Some probability distributions in modeling DNA replication. Ann. Appl. Prob. 13, 12071230.

[6] Piau, D. (2000). Quasi-renewal estimates. J. Appl. Prob. 37, 269-275, 1171-1172. 